\title{
Dilution of axion dark radiation
}

\section{Hironori Hattori, Tatsuo Kobayashi, Naoya Omoto}

Department of Physics, Hokkaido University, Sapporo 060-0810, Japan

\section{Osamu Seto*}

Department of Life Science and Technology, Hokkai-Gakuen University, Sapporo 062-8605,

Japan

E-mail: seto@physics.umn.edu

The Peccei-Quinn (PQ) mechanism provide a promising solution to the strong $\mathrm{CP}$ problem in the standard model of particle physics. Axion appears as its pseudo-Nambu-Goldstone boson of the PQ symetry breaking. Coherently generated PQ scalar fields could dominate the energy density in the early Universe and decay into relativistic axions, which would conflict with the current dark radiation constraints. We show that a thermal inflation driven by a $U(1)$ gauged Higgs field dilutes such axions. We discuss an available baryogenesis mechanism for the $U(1)_{B-L}$ gauge symmetry.

The European Physical Society Conference on High Energy Physics 22-29 July 2015

Vienna, Austria

${ }^{*}$ Speaker. 


\section{Introduction}

The standard model (SM) for elementary particles has been successful in describing high energy phenomena at colliders. The strong $\mathrm{CP}$ problem is one of long standing questions concerning the SM. A mechanism proposed by Peccei and Quinn [1] with introducing global $U(1)$ symmetry, Peccei-Quinn (PQ) symmetry, elegantly solves this problem. The original model had been excluded by the experimental results. However, so-called invisible axion models $[2,3]$ are promising and viable models. As a result of the global $U(1)$ PQ symmetry breaking, axion field, which is its Nambu-Goldstone (NG) boson and becomes a pseudo-NG boson due to the QCD anomaly, appears.

Cosmology based on particle theory with the PQ symmetry would be interesting but not so simple. One appealing feature is, as is well-known, axion is a promising candidate for dark matter in our Universe [4]. On the other hand, the evolution of the early Universe would be nontrivial as follows. The PQ scalar field could be produced as coherent oscillation due to its scalar nature and temporally dominate the energy density of the Universe if its decay rate is very small because of suppressed couplings. The radial direction of the PQ scalar ${ }^{1}$ would mostly decay into axions. Those overproduced massless axions act as dark radiation which is nowadays stringently constrained [5].

Thermal inflation is a well-known mechanism to dilute unwanted relics [6] and is driven by a scalar field $\varphi$, often refered to as the flaton. We show the condition of successful thermal inflation driven by a gauged $U(1)$ Higgs field to dilute axions generated by the late decay of the dominated PQ scalar field [7]. If this flaton $\varphi$ is a gauge singlet and has an (approximate) global $U(1)$, then the axions associated with the flaton could be produced again as shown in Ref. [8]. In order to avoid this problem, we assume that a flaton field is charged under a local $U(1)$ symmetry. We also discuss the implication in the case that this local $U(1)$ symmetry is identified with gauged $U(1)_{B-L}$ [9].

\section{Thermal inflation in an axion-dominated Universe}

We consider the scalar potential of the flaton $\varphi$ as

$$
V(\varphi)=V_{0}-m^{2}|\varphi|^{2}+\frac{|\varphi|^{2 n}}{\Lambda^{2(n-2)}}
$$

A flaton field $\varphi$ is suppposed to be in thermal equilibrium through interactions with particles in the hot thermal bath. Then, the thermal correction; in fact, the thermal mass term,

$$
\delta V=\frac{h^{2}}{24} T^{2}|\varphi|^{2},
$$

with $T$ being the temperature of the thermal plasma, appears in the scalar potential. Here, $h^{2}$ is parametrizing the coefficient.

The resultant number of $e$-fold in the axion-dominated Universe is estimated as

$$
\begin{aligned}
N_{2 n}= & -\ln 4 \sqrt{3}-\frac{1}{4} \ln \left(\frac{\pi^{2}}{30} g_{*}\right)+\frac{1}{2} \ln \frac{\Lambda}{M_{P}} h \\
& -\frac{1}{4} \ln \frac{n^{2}}{4(n-1)}+\frac{1}{2}(n-2) \ln \left(\frac{M_{P}}{v}\right),
\end{aligned}
$$

\footnotetext{
${ }^{1}$ From now on, we simply call it the PQ scalar.
} 
with $M_{P}$ being the reduced Planck mass. We list various physical quantities in Table 1.

Table 1: Quantities in thermal inflation

\begin{tabular}{|c|c|c|c|c|c|c|c|}
\hline$\Lambda(\mathrm{GeV})$ & $h$ & $v(\mathrm{GeV})$ & $T_{i}(\mathrm{GeV})$ & $T_{f}(\mathrm{GeV})$ & $N$ & $\Delta N_{e f f}$ & $T_{R}(\mathrm{GeV})$ \\
\hline $10^{16}$ & $8.27 \times 10^{-3}$ & $10^{8}$ & $2.79 \times 10^{3}$ & $1.03 \times 10^{3}$ & 1.00 & 0.05 & $5.9 \times 10^{3}$ \\
\hline $10^{16}$ & $8.27 \times 10^{-2}$ & $10^{10}$ & $2.79 \times 10^{6}$ & $1.03 \times 10^{6}$ & 1.00 & 0.05 & $5.9 \times 10^{6}$ \\
\hline $10^{16}$ & $8.27 \times 10^{-1}$ & $10^{12}$ & $2.87 \times 10^{9}$ & $1.03 \times 10^{9}$ & 1.00 & 0.05 & $5.9 \times 10^{9}$ \\
\hline
\end{tabular}

\section{Relic abundances}

\subsection{Axion dark radiation}

If the PQ scalar field dominates the energy density of the Universe, its decay produces many axions, and the Universe bedomes relativistic axion dominated. When the total energy density $\rho_{\text {total }}$ from dominated axion $\rho_{a}$ and subdominant radiation $\rho_{\text {rad }}$ becomes comparable with $V(\varphi), t=t_{i}$, the thermal inflation begins. After the thermal inflation, $\varphi$ decays into SM particles and potentially non-SM particles again. The resultant axion dark radiation contribution is estimated in terms of $\Delta N_{e f f}$ as

$$
\Delta N_{e f f}=\frac{43}{7}\left(\frac{43 / 4}{g_{*}}\right)^{1 / 3} \times\left.\frac{\rho_{a}}{\rho_{\text {rad }}}\right|_{H=\Gamma} .
$$

\subsection{Reheating temperature and possible baryogenesis scenarios}

We adopt the reheating temperature after thermal inflation $T_{R}$ under the assumption of the instantaneous reheating $\Gamma=H\left(t_{f}\right)$, which gives the highest reheating temperature. Available baryogenesis mechanisms depend on $T_{R}$.

For $T_{R} \gtrsim 10^{9} \mathrm{GeV}$, thermal leptogenesis by the lightest heavy RH neutrino decay of those with hierarchical masses is one of the simplest scenarios of baryogenesis [10,11].

Nonthermal leptogenesis by RH neutrinos with hierarchical masses is available for a reheating temperature $10^{9} \mathrm{GeV} \gtrsim T_{R} \gtrsim 10^{6} \mathrm{GeV}$ [12]. If this local $U(1)$ is in fact the gauged $U(1)_{B-L}$ symmetry, $\varphi$ is identified with the Higgs field to break this symmetry with the $B-L$ charge 2 , and the decay of $\varphi$ into two RH neutrinos $N_{R}$ is nothing but nonthermal production of $N_{R}$.

For $T_{R} \lesssim 10^{6} \mathrm{GeV}$, low-scale thermal leptogenesis requires an enhancement of CP violation. Here, for information, we refer two examples. One is the so-called resonant leptogenesis, where two RH neutrino masses are strongly degenerated and CP violation is enlarged due to RH neutrino self-energy [13]. Another way is an extension of the Higgs sector, e.g., neutrinophilic Higgs model [14]. Another promising scenario would be electroweak baryogenesis [15].

\subsection{Results}

We summarize the viable parameter space and available baryogenesis mechanism for some benchmark points. In order to have a large enough CP violation $\varepsilon \gtrsim 10^{-6}$ in the $N_{R}$ decay, we take $M_{N_{R}} \simeq 10^{9} \mathrm{GeV}[16,17]$ and that the decay $\varphi \rightarrow N_{R} N_{R}$ is kinematically forbidden for $m_{\varphi}<10^{9}$ $\mathrm{GeV}$. We consider two cases of the PQ scalar VEV, $v=10^{10}$ and $10^{12} \mathrm{GeV}$. We note that, for baryogenesis, the conclusion is the same for $v \lesssim 10^{10} \mathrm{GeV}$. 


\subsection{1 $n=3, v=10^{12} \mathbf{G e V}$ case}

For most of the parameter space, we have $T_{R}>10^{9} \mathrm{GeV}$. The thermal leptogenesis could work.

\subsection{2 $n=3, v=10^{10} \mathbf{G e V}$ case}

$T_{R}>10^{6} \mathrm{GeV}$ is realized; however, $m_{\varphi} \lesssim 10^{9} \mathrm{GeV}$. Nonthermal leptogenesis by the $\varphi$ decay does not work because the $\varphi$ decay is kinematically forbidden. A low-scale thermal leptogenesis with an enhanced CP violation or the electroweak baryogenesis with the extension of the Higgs sector is needed.
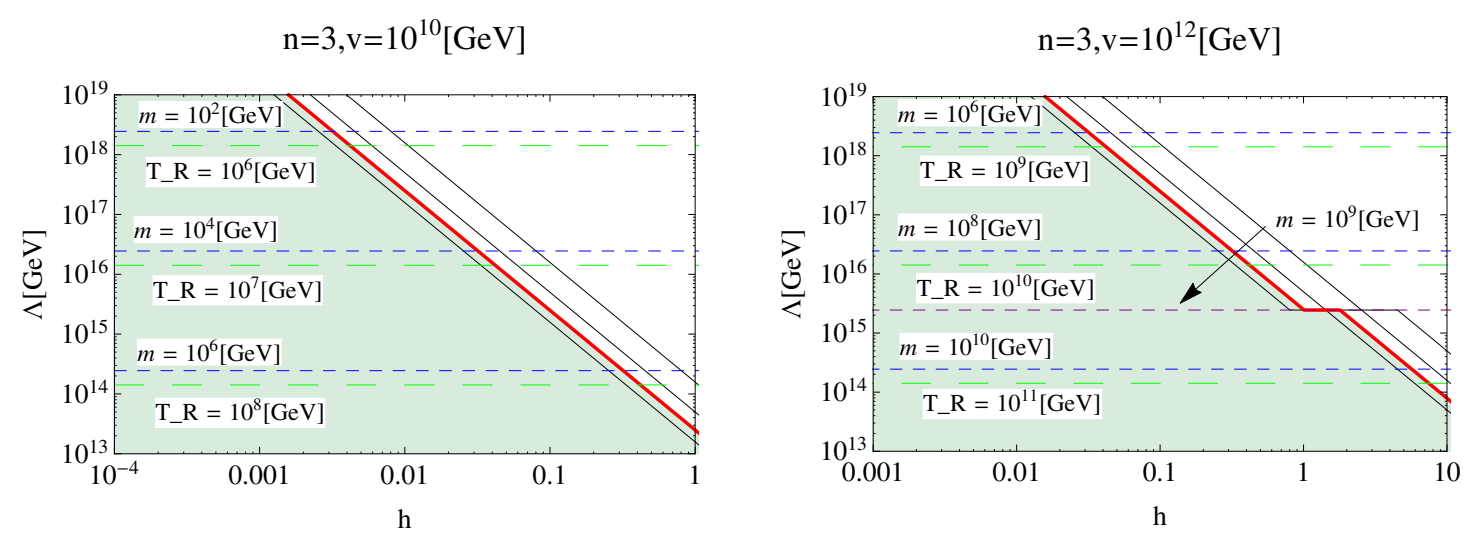

Figure 1: Contours of the resultant $\Delta N_{\text {eff }}=1,0.4$ (thick red), 0.1 , and 0.01 with solid lines from left to right, the mass of $\varphi$ with dashed lines and the possible maximal reheating temperature after thermal inflation $T_{R}$ with long dashed lines. The shaded region corresponds to $\Delta N_{e f f}>0.4$ which is disfavored by the Planck (2015) data.

\section{Summary}

We have investigated scenarios with successful thermal inflation by a gauged $U(1)$ Higgs flaton field to dilute axions generated by late decay of the dominated PQ scalar field. If this $U(1)$ symmetry is the gauged $U(1)_{B-L}$, high- or low-scale thermal leptogenesis or the electroweak baryogenesis is an available baryogenesis scenario, depending on parameters.

\section{Acknowledgments}

This work was supported in part by the Grant-in-Aid for Scientific Research No. 25400252 (T.K.) and on Innovative Areas No. 26105514 (O.S.) from the Ministry of Education, Culture, Sports, Science and Technology in Japan.

\section{References}

[1] R. D. Peccei and H. R. Quinn, Phys. Rev. Lett. 38, 1440 (1977).

[2] J. E. Kim, Phys. Rev. Lett. 43103 (1979);

M. A. Shifman, A. I. Vainshtein and V. I. Zakharov, Nucl. Phys. B 166, 493 (1980). 
[3] M. Dine, W. Fischler and M. Srednicki, Phys. Lett. B 104, 199 (1981);

A. R. Zhitnitsky, Sov. J. Nucl. Phys. 31, 260 (1980) [Yad. Fiz. 31, 497 (1980)].

[4] For a review, see, e.g., M. Kawasaki and K. Nakayama, Ann. Rev. Nucl. Part. Sci. 63, 69 (2013).

[5] P. A. R. Ade et al. [Planck Collaboration], arXiv:1502.01589 [astro-ph.CO].

[6] D. H. Lyth and E. D. Stewart, Phys. Rev. Lett. 75, 201 (1995);

D. H. Lyth and E. D. Stewart, Phys. Rev. D 53, 1784 (1996).

[7] H. Hattori, T. Kobayashi, N. Omoto and O. Seto, Phys. Rev. D 92, 023517 (2015).

[8] T. Asaka and M. Kawasaki, Phys. Rev. D 60, 123509 (1999).

[9] R. N. Mohapatra and R. E. Marshak, Phys. Rev. Lett. 44, 1316 (1980) Erratum [Phys. Rev. Lett. 44, 1644 (1980)];

R. E. Marshak and R. N. Mohapatra, Phys. Lett. B 91, 222 (1980).

[10] M. Fukugita and T. Yanagida, Phys. Lett. B 174, 45 (1986).

[11] For a review, see, e.g., W. Buchmuller, P. Di Bari and M. Plumacher, Annals Phys. 315, 305 (2005).

[12] T. Asaka, K. Hamaguchi, M. Kawasaki and T. Yanagida, Phys. Lett. B 464, 12 (1999).

[13] A. Pilaftsis and T. E. J. Underwood, Nucl. Phys. B 692, 303 (2004).

[14] N. Haba and O. Seto, Prog. Theor. Phys. 125, 1155 (2011); Phys. Rev. D 84, 103524 (2011).

[15] For a review, see, e.g., D. E. Morrissey and M. J. Ramsey-Musolf, New J. Phys. 14, 125003 (2012).

[16] W. Buchmuller, P. Di Bari and M. Plumacher, Nucl. Phys. B 643, 367 (2002) [Nucl. Phys. B 793, 362 (2008)].

[17] S. Davidson and A. Ibarra, Phys. Lett. B 535, 25 (2002). 